\title{
On Neighborhood Degree-Based Topological Analysis of Polyphenylene Network
}

\author{
Chuang Sun, ${ }^{1}$ A. Khalid, ${ }^{2}$ H. M. Usman, ${ }^{2}$ A. Ahmad, ${ }^{2}$ M. K. Siddiqui, ${ }^{3}$ and S. A. Fufa $\mathbb{D}^{4}$ \\ ${ }^{1}$ School of Management, WuHan Polytechnic University, Wuhan 430048, China \\ ${ }^{2}$ Department of Mathematics, Air University Multan Campus, Multan, Pakistan \\ ${ }^{3}$ Department of Mathematics, COMSATS University Islamabad, Lahore Campus, Lahore, Pakistan \\ ${ }^{4}$ Department of Mathematics, Addis Ababa University, Addis Ababa, Ethiopia
}

Correspondence should be addressed to S. A. Fufa; samuel.asefa@aau.edu.et

Received 31 December 2021; Accepted 2 February 2022; Published 21 February 2022

Academic Editor: Alessandro Lo Schiavo

Copyright $\odot 2022$ Chuang Sun et al. This is an open access article distributed under the Creative Commons Attribution License, which permits unrestricted use, distribution, and reproduction in any medium, provided the original work is properly cited.

\begin{abstract}
Organic compounds such as polyphenylene are very important and useful for the synthesis of many new organic compounds due to their physio-chemical properties. To ascertain these properties, one can use QSPR/QSAR methods which necessitate the computation of topological indices. The topological indices based on two newly introduced abstract notions of ev-degree and vedegree are in practice to model numerous chemical properties as well as physical properties of organic, inorganic, hybrid, and biological compounds. In this study, we computed a certain number of topological indices for the chemical graph of polyphenylene network which will help to model some of its physio-chemical properties.
\end{abstract}

\section{Introduction}

The detailed critical inspection in order to discover essential features or meanings of chemical compounds graphically is known as chemical graph theory. It is the branch of mathematics, which alloys chemistry and graph theory. In graph theory, a simple graph or just graph $G(V, E)$ is constructed by two sets: $V=\left\{v_{1}, \ldots, v_{n}\right\}$, the set of vertices, and $E=\left\{e_{1}, \ldots, e_{m}\right\}$, the set of edges. Each $v \in V$ represents a node in the graph and each $e \in E$ denotes the line joining two nodes.

In chemical graph theory, the image obtained from diffraction of X-rays or electron microscopy of a compound (biological or chemical) is drawn into plane and lighted upon its symmetry, and then, peculiarities of this compound is mathematically modeled. The simple sketch of the image of compound is known as the chemical graph where we assume that the ends or vertices are atoms and lines or edges are the bonds between the atoms. Chemical graph theory helps to understand different properties, namely, molecular structure, kinetics of molecules, atoms or electrons, chain or patterns of polymers, crystals and clusters, aromaticity, nuclear magnetic resonance (NMR) analysis, depicting orbitals, and electrons behaviфrs. Ante Graovac, Alexandru Balaban, Haruo Hosoya, Iv a $n$ Gutman, Nenad Trinajstic, and Milan Randic are few scientists who introduced graph theory in chemistry [1].

The job of mathematical modeling the properties of chemical compounds is done by topological indices which we define as a number obtained by a real-valued function, $g=g(e), g=g(v)$ or $g=g(e, v)$, that is applied to any chemical graph (or molecular structure) of a compound to determine its topology, is known as topological graph index or just topological index (plural: topological indices), where $e$ and $v \in \mathbb{Z}^{+}$are edges and vertices of graph, for example, Zagreb indices and their variants, distance indices, detour index, and Wiener index. There are different kinds of topological indices based on degree, distance, and counting [2]. Many physical and chemical properties of different chemical and biological compounds have been modeled mathematically by the aid of topological indices such as boiling point, anti-leishmanial effect, acute toxicity, radial scavenging activity, and many more [3-5]. In this study, we considered some topological indices based on degree of 
vertices and edges, ve-degree of vertices, and ev-degree of edges. To understand the terms used in formulas of topological indices, first, we consider the following basic definitions [6-8].

For a graph $X(V, E)$, we have

(1) Degree of a vertex $v$ means the number of edges connected to the vertex $v$ denoted by $\mathrm{d}_{X}(v)$

(2) The number $\mathrm{d}_{X}(v)+\mathrm{d}_{X}(w)-2$ is called degree of edge $e$ if $e$ is formed by joining the vertex $v$ and $w$, denoted by $\mathrm{d}_{X}(e)$

(3) The set $N(w)=\{v \in V: v$ and $w$ are nodes of some edge $e \in E\}$ is called the open neighborhood of the vertex $w$

(4) The set $\{w\} \cup N(w)$ is the closed neighborhood of $w$ denoted by $N[w]$

(5) The number of nonidentical edges that are incident to each vertex in the closed neighbourhood of the vertex $v$ is the ve-degree of vertex $v$ denoted by $d_{X}^{v e} v$ [9]

(6) If $v$ and $w$ are nodes of an edge $e$, then the order of $N[v] \cup N[w]$ is equal to the ev-degree [9] of edge $e$, denoted by $d_{X}^{e v}(e)$

For a graph $X(V, E)$, the topological indices under consideration are given below:

(i) Randić index [10]:

$$
R(X)=\sum_{e=1}^{|E|} \frac{1}{\sqrt{d_{X}(u) \times d_{X}(v)}} .
$$

(ii) $e v$-degree Randić index [11]:

$$
R^{e v}(X)=\sum_{e=1}^{|E|} \frac{1}{\sqrt{d_{X}^{e v}(e)}} .
$$

(iii) ve-degree Randić index:

$$
R^{v e}(X)=\sum_{e=1}^{|E|} \frac{1}{\sqrt{d_{X}^{v e}(u) \times d_{X}^{v e}(v)}} .
$$

(iv) Reciprocal ve-degree Randić index [12]:

$$
R R^{e v}(H)=\sum_{e=1}^{|E|} d_{X}^{e v}(e)^{1 / 2}
$$

Milan Randić, a chemist, introduced the "branching index" in 1975 as a topological index $R$ for evaluating the degree of branching in the carbon-atom skeleton of saturated hydrocarbons [10]. Moreover, it is also used to model the cavity surface area of different alcohols. In [11], Suleyman Ediz introduced $e v$-degree Randić (2) index and proved that it gives more accurate correlation than the previous one.

First ve-degree Zagreb beta index:

$$
M_{i}^{\mathrm{bve}}(X)=\sum_{e=1}^{|E|}\left(d_{X}^{v e} v+d_{X}^{v e} w\right)
$$

Second ve-degree Zagreb index:

$$
M_{i i}^{v e}(X)=\sum_{e=1}^{|E|}\left(d_{X}^{v e} v \times d_{X}^{v e} w\right)
$$

Redefined third ve-degree Zagreb index [13]:

$$
\operatorname{RZG}_{i i i}^{v e}(H)=\sum_{e=1}^{|E|}\left(d_{X}^{v e}(u) \times d_{X}^{v e}(v)\right)\left(d_{X}^{v e}(u)+d_{X}^{v e}(v)\right) \text {. }
$$

The modified ev-degree Zagreb index [12]:

$$
{ }^{*} M^{e v}(H)=\sum_{e=1}^{|E|} \frac{1}{d_{X}^{e v}(e)^{2}} .
$$

Zagreb indices were first used to model anti-inflammatory agility in different acids [14], and then, $M_{1}$ and $M_{2}$ were used to model the clearance of cephalosporins and fraction bound in humans [15].

(i) ve-degree sum-connectivity index:

$$
v e-S C(X)=\sum_{e=1}^{|E|} \frac{1}{\sqrt{d_{X}^{v e}(u)+d_{X}^{v e}(v)}} .
$$

(ii) ve-degree atom-bond connectivity index:

$$
v e-\operatorname{ABC}(X)=\sum_{e=1}^{|E|} \sqrt{\frac{d_{X}^{v e}(u)+d_{X}^{v e}(v)-2}{d_{X}^{v e}(u) \times d_{X}^{v e}(v)}} .
$$

Atom-bond connectivity index is used to model heat of formation $\Delta H_{f}$ in alkanes [16].

(iii) ve-degree geometric arithmetic index:

$$
v e-G A(X)=\sum_{e=1}^{|E|} \frac{2 \sqrt{d_{X}^{v e}(u) \times d_{X}^{v e}(v)}}{d_{X}^{v e}(u)+d_{X}^{v e}(v)} .
$$

(iv) Arithmetic-geometric index [17]:

$$
v e-A G(X)=\sum_{e=1}^{|E|} \frac{d_{X}^{v e}(u)+d_{X}^{v e}(v)}{2 \sqrt{d_{X}^{v e}(u) \times d_{X}^{v e}(v)}} .
$$

In [18], the geometrical-arithmetic index (GA) was introduced and used to model the following properties of chemical compounds:

(i) Acentric factor (AcenFac)

(ii) Boiling point (BP)

(iii) Entropy (S)

(iv) Enthalpy of formation (HFORM)

(v) Enthalpy of vaporization (HVAP) 


\section{(vi) Standard enthalpy of vaporization (DHVAP)}

Recently, some other topological indices (listed below) are introduced on the basis of ev- and ve-degree to model chemical properties written above which give better correlation coefficients of about 0.99088 [19].

(i) ve-degree harmonic index:

$$
H^{v e}(X)=\sum_{e=1}^{|E|} \frac{2}{d_{X}^{v e}(u)+d_{X}^{v e}(v)} .
$$

(ii) $e v$-degree inverse index [12]:

$$
I D^{e v}(H)=\sum_{e=1}^{|E|} \frac{1}{d_{X}^{e v}(e)} .
$$

(iii) ve-degree inverse sum index [20]:

$$
\operatorname{ISI}^{v e}(H)=\sum_{e=1}^{|E|} \frac{d_{X}^{v e}(u) \times d_{X}^{v e}(v)}{d_{X}^{v e}(u)+d_{X}^{v e}(v)} .
$$

(iv) F-ev-degree index [12]:

$$
F^{e v}(H)=\sum_{e=1}^{|E|} \mathrm{d}_{X}^{e v}(e)^{3} .
$$

(v) F-ve-degree index [21]:

$$
F^{v e}(H)=\sum_{e=1}^{|E|}\left(d_{X}^{v e}(u)^{2}+d_{X}^{v e}(v)^{2}\right) .
$$

(vi) First hyper-ve-degree index [21]:

$$
H M_{1}^{v e}(H)=\sum_{e=1}^{|E|}\left(d_{X}^{v e}(u)+d_{X}^{v e}(v)\right)^{2} .
$$

(vii) Second hyper-ve-degree index [21]:

$$
H M_{2}^{v e}(H)=\sum_{e=1}^{|E|}\left(d_{X}^{v e}(u) \times d_{X}^{v e}(v)\right)^{2} \text {. }
$$

For more details, see[22-24].

\section{Polyphenylene Network}

Hydrocarbons, which are organic compounds formulated completely by the atoms of hydrogen and carbon elements, are one of the world's most prominent sources of energy and are found mostly in petroleum form, natural gas, and crude oil. The majority of hydrocarbons are linear chains, rings, or a mix of the two. Polyphenylene, a two-dimensional (2D) limitless hydrocarbon with the formula $\mathrm{C}_{6} \mathrm{H}_{4}$, that may be formed experimentally, is thermodynamically stable. Furthermore, due to its new structural features, 2D polyphenylene could be used in a variety of industries, such as a barrier for $\mathrm{H}_{2}$ purification. When $\mathrm{H}_{2}$ is produced by the conventional process of steam methane reformation, certain less desirable species are produced, such as $\mathrm{CO}, \mathrm{CO}_{2}$, and $\mathrm{CH}_{4}$. As a result, isolating $\mathrm{H}_{2}$ from these species is critical for its storage and use [25]. SRP is a superb option for many demanding applications including semiconductor components, high-performance bearings, bushings, valves, valve seats, and aircraft substructures, due to its remarkable mechanical, chemical, thermal, and electrical qualities. SRP is an ideal contender for light-weight high-performance applications due to its high specific strength. Antistatic coatings made of electrically conductive polyphenylene (p- or n-doped) are used to protect integrated circuits from static charges, humidity, and corrosion [26]. The following are important performance characteristics:

Mechanical stiffness and strength are extremely high. High compression strength and resistance to pressure, excellent wear and scratch resistance, and good cold temperature stability (to around $-270^{\circ} \mathrm{C}$ ), $155^{\circ} \mathrm{C}$ is a high glass transition temperature. Before and after processing, there is exceptional dimensional stability, low coefficient of thermal expansion (low thermal shrinkage), excellent acid, and basic resistance. With resistance to solvents and hot steam (but lower than PEEK), processability is good (can be extruded and injection molded).

2.1. Mathematical Work and Discussion. In this section, we will compute and discuss all the topological indices mentioned before, for the graph of polyphenylene network PN, as shown in Figure 1, where, in Figure 2, we show the unit segment of the graph. In the graph, we have vertices joined with single edge, double edges, and triple edges (i.e., $\left.\mathrm{d}_{\mathrm{PN}}(v) \in\{1,2,3\}\right)$. In the graph of polyphenylene network $\mathrm{PN}(V, E)$, we observe that there are $30 m^{2}+8 m-4$ vertices and $34 m^{2}+m+3$ edges. More information about the graph is given in Table 1 .

$$
\begin{gathered}
(a) \text { ve }-\mathrm{SC}(\mathrm{PN})=\left(3 \sqrt{3}+\frac{5}{2 \sqrt{2}}+\frac{7}{\sqrt{10}}+\frac{4}{\sqrt{15}}\right) m^{2}+\frac{m}{2 \sqrt{3}}-\frac{1}{2 \sqrt{2}}+\frac{1}{2 \sqrt{3}}+\frac{1}{\sqrt{10}}+\frac{2}{\sqrt{15}} \\
(b) \mathrm{ve}-\mathrm{ABC}(\mathrm{PN})=\left(\frac{14 \sqrt{2}}{5}+\sqrt{10}+18 \sqrt{2 / 7}+\sqrt{26 / 7}\right) m^{2}+\sqrt{2 / 7} m+\frac{2 \sqrt{2}}{5}-\sqrt{2 / 5}+\sqrt{2 / 7}+\sqrt{13 / 14}
\end{gathered}
$$

Theorem 1. Let $P N(V, E)$ be the graph of polyphenylene network; then, 
<smiles>Cc1ccc(-c2cc(-c3ccc(C)cc3)c(-c3ccc(-c4cc(-c5ccc(C)cc5)c(-c5ccc(-c6cc(-c7ccc(C)cc7)c(-c7ccc(C)cc7)cc6-c6cc(-c7ccc(C)cc7)c(-c7ccc(C)cc7)cc6-c6ccc(C)cc6)cc5)cc4-c4ccc(-c5cc(-c6cc(-c7ccc(C)cc7)c(-c7ccc(C)cc7)cc6-c6ccc(C)cc6)c(-c6ccc(C)cc6)cc5-c5ccc(C)cc5)cc4)cc3)cc2-c2ccc(C)cc2)cc1</smiles>

FIGURE 1: Molecular graph of polyphenylene network.<smiles>Cc1ccc(-c2cc(-c3ccc(C)cc3)c(-c3ccc(C)cc3)cc2-c2ccc(C)cc2)cc1</smiles>

FIgURE 2: Unit of the graph of polyphenylene network.

TABLE 1: Edge partition table of polyphenylene network.

\begin{tabular}{lcccc}
\hline$\left(d_{P N}(u), d_{P N}(v)\right)$ & $\mathrm{d}_{\mathrm{PN}}(e)$ & $\left(d_{P N}^{v e}(u), d_{P N}^{v e}(v)\right)$ & $d_{P N}^{e v}(e)$ & Frequency \\
\hline$(1,3)$ & 2 & $(3,5)$ & 4 & $5 m^{2}-1$ \\
$(2,2)$ & 2 & $(5,5)$ & 4 & $7 m^{2}+1$ \\
$(2,3)$ & 3 & $(5,7)$ & 5 & 6 \\
$(3,3)$ & 4 & $(7,8)$ & 6 & $18 m^{2}+m^{2}+1$ \\
\hline
\end{tabular}

(a) $R(P N)=\frac{1}{6}(10 \sqrt{3}+18 \sqrt{6}+29) m^{2}+\frac{m}{\sqrt{6}}+\frac{1}{6}(-2 \sqrt{3}+\sqrt{6}+7)$,

(b) $R^{e v}(P N)=\left(2 \sqrt{2 / 3}+\frac{18}{\sqrt{5}}+6\right) m^{2}+\frac{m}{\sqrt{5}}+\sqrt{2 / 3}+\frac{1}{\sqrt{5}}$,

(c) $R^{v e}(P N)=\left(\sqrt{5 / 3}+\sqrt{2 / 7}+\frac{7}{5}+\frac{18}{\sqrt{35}}\right) m^{2}+\frac{m}{\sqrt{35}}+\frac{1}{5}+\frac{1}{\sqrt{14}}-\frac{1}{\sqrt{15}}+\frac{1}{\sqrt{35}}$,

(d) $R R^{e v}(P N)=2(9 \sqrt{5}+2 \sqrt{6}+12) m^{2}+\sqrt{5} m+\sqrt{5}+2 \sqrt{6}$.

Proof. To prove (a), we expand equation (1) and substitute values from Table 1, which gives 


$$
\begin{aligned}
R(P N) & =\frac{4 m^{2}+2}{\sqrt{3 \times 3}}+\frac{5 m^{2}-1}{\sqrt{1 \times 3}}+\frac{18 m^{2}+m+1}{\sqrt{2 \times 3}}+\frac{7 m^{2}+1}{\sqrt{2 \times 2}} \\
& =\frac{1}{3}\left(4 m^{2}+2\right)+\frac{1}{2}\left(7 m^{2}+1\right)+\frac{5 m^{2}-1}{\sqrt{3}}+\frac{18 m^{2}+m+1}{\sqrt{6}} \\
& =\frac{1}{6}\left((10 \sqrt{3}+18 \sqrt{6}+29) m^{2}+\sqrt{6} m-2 \sqrt{3}+\sqrt{6}+7\right) \\
& =\frac{1}{6}(10 \sqrt{3}+18 \sqrt{6}+29) m^{2}+\frac{m}{\sqrt{6}}+\frac{1}{6}(-2 \sqrt{3}+\sqrt{6}+7) .
\end{aligned}
$$

To prove (b), we expand (2) and substitute values from Table 1, which gives

$$
\begin{aligned}
R^{e v}(P N) & =\frac{4 m^{2}+2}{\sqrt{6}}+\frac{5 m^{2}-1}{\sqrt{4}}+\frac{18 m^{2}+m+1}{\sqrt{5}}+\frac{7 m^{2}+1}{\sqrt{4}} \\
& =\frac{4 m^{2}+2}{\sqrt{6}}+\frac{1}{2}\left(5 m^{2}-1\right)+\frac{1}{2}\left(7 m^{2}+1\right)+\frac{18 m^{2}+m+1}{\sqrt{5}} \\
& =\left(2 \sqrt{2 / 3}+\frac{18}{\sqrt{5}}+6\right) m^{2}+\frac{m}{\sqrt{5}}+\sqrt{2 / 3}+\frac{1}{\sqrt{5}} .
\end{aligned}
$$

To prove (c), we expand (3) and substitute values from Table 1, which gives

$$
\begin{aligned}
R^{v e}(P N)= & \frac{4 m^{2}+2}{\sqrt{7 \times 8}}+\frac{5 m^{2}-1}{\sqrt{3 \times 5}}+\frac{18 m^{2}+m+1}{\sqrt{5 \times 7}}+\frac{7 m^{2}+1}{\sqrt{5 \times 5}} \\
= & \frac{2 m^{2}+1}{\sqrt{14}}+\frac{1}{5}\left(7 m^{2}+1\right)+\frac{5 m^{2}-1}{\sqrt{15}}+\frac{18 m^{2}+m+1}{\sqrt{35}} \\
= & \left(\sqrt{5 / 3} \sqrt{2 / 7}+\frac{7}{5}+\frac{18}{\sqrt{35}}\right) m^{2}+\frac{m}{\sqrt{35}}+\frac{1}{5}+\frac{1}{\sqrt{14}} \\
& -\frac{1}{\sqrt{15}}+\frac{1}{\sqrt{35}} .
\end{aligned}
$$

To prove (d), we expand (4) and substitute values from Table 1, which gives

$$
\begin{aligned}
R R^{e v}(P N) & =\sqrt{6}\left(4 m^{2}+2\right)+\sqrt{4}\left(5 m^{2}-1\right)+\sqrt{5}\left(18 m^{2}+m+1\right)+\sqrt{4}\left(7 m^{2}+1\right) \\
& =2\left(5 m^{2}-1\right)+2\left(7 m^{2}+1\right)+\sqrt{5}\left(18 m^{2}+m+1\right)+\sqrt{6}\left(4 m^{2}+2\right) \\
& =2(9 \sqrt{5}+2 \sqrt{6}+12) m^{2}+\sqrt{5} m+\sqrt{5}+2 \sqrt{6} .
\end{aligned}
$$

Theorem 2. Let $P N(V, E)$ be the graph of polyphenylene network; then,
(a) $M_{i}^{\text {bve }}(P N)=386 m^{2}+12 m+44$,
(b) $M_{i i}^{v e}(P N)=1104 m^{2}+35 m+157$,
(c) $R Z G_{i i i}^{v e}(P N)=10\left(223+42 m+1327 m^{2}\right)$,
$(d){ }^{*} M^{e v}(P N)=\frac{1}{900}\left(1423 m^{2}+36 m+86\right)$.

$$
\begin{aligned}
M_{i}^{\text {bve }}(P N) & =(7+8)\left(4 m^{2}+2\right)+(3+5)\left(5 m^{2}-1\right)+(5+7)\left(18 m^{2}+m+1\right) \\
& =+(5+5)\left(7 m^{2}+1\right) 15\left(4 m^{2}+2\right)+8\left(5 m^{2}-1\right)+10\left(7 m^{2}+1\right)+12\left(18 m^{2}+m+1\right) \\
& =386 m^{2}+12 m+44
\end{aligned}
$$

Proof. To prove (a), expand equation (5) and substitute values from Table 1, which gives

To prove (b), expand (6) and substitute values from Table 1, which gives 


$$
\begin{aligned}
M_{i i}^{v e}(P N) & =(7 \times 8)\left(4 m^{2}+2\right)+(3 \times 5)\left(5 m^{2}-1\right)+(5 \times 7)\left(18 m^{2}+m+1\right)+(5 \times 5)\left(7 m^{2}+1\right) \\
& =56\left(4 m^{2}+2\right)+15\left(5 m^{2}-1\right)+25\left(7 m^{2}+1\right)+35\left(18 m^{2}+m+1\right) \\
& =1104 m^{2}+35 m+157 .
\end{aligned}
$$

To prove (c), expand (7) and substitute values from Table 1, which gives

$$
\begin{aligned}
\mathrm{RZG}_{i i i}^{v e}(P N) & =\left(5 m^{2}-1\right)(3 \times 5)(3+5)+\left(7 m^{2}+1\right)(5 \times 5)(5+5)+\left(18 m^{2}+m+1\right)(5 \times 7)(5+7)+\left(4 m^{2}+2\right)(7 \times 8)(7+8) \\
& =840\left(2+4 m^{2}\right)+120\left(-1+5 m^{2}\right)+250\left(1+7 m^{2}\right)+420\left(1+m+18 m^{2}\right) \\
& =10\left(223+42 m+1327 m^{2}\right) .
\end{aligned}
$$

To prove (d), expand (8) and substitute values from Table 1, which gives

$$
\begin{aligned}
* M^{e v}(P N) & =\left(5 m^{2}-1\right) \times \frac{1}{4^{2}}+\left(7 m^{2}+1\right) \times \frac{1}{4^{2}}+\left(18 m^{2}+m+1\right) \times \frac{1}{5^{2}}+\left(4 m^{2}+2\right) \times \frac{1}{6^{2}} \\
& =\frac{1}{36}\left(4 m^{2}+2\right)+\frac{1}{16}\left(5 m^{2}-1\right)+\frac{1}{16}\left(7 m^{2}+1\right)+\frac{1}{25}\left(18 m^{2}+m+1\right) \\
& =\frac{1}{900}\left(1423 m^{2}+36 m+86\right) .
\end{aligned}
$$

Theorem 3. Let $P N(V, E)$ be the graph of polyphenylene network; then,
Proof. To prove (a), expanding equation (9) and substituting values from Table 1 , we find

$$
\begin{aligned}
v e-S C(P N) & =\frac{4 m^{2}+2}{\sqrt{7+8}}+\frac{5 m^{2}-1}{\sqrt{3+5}}+\frac{18 m^{2}+m+1}{\sqrt{5+7}}+\frac{7 m^{2}+1}{\sqrt{5+5}} \\
& =\frac{4 m^{2}+2}{\sqrt{15}}+\frac{5 m^{2}-1}{2 \sqrt{2}}+\frac{18 m^{2}+m+1}{2 \sqrt{3}}+\frac{7 m^{2}+1}{\sqrt{10}} \\
& =\left(3 \sqrt{3}+\frac{5}{2 \sqrt{2}}+\frac{7}{\sqrt{10}}+\frac{4}{\sqrt{15}}\right) m^{2}+\frac{m}{2 \sqrt{3}}-\frac{1}{2 \sqrt{2}}+\frac{1}{2 \sqrt{3}}+\frac{1}{\sqrt{10}}+\frac{2}{\sqrt{15}} .
\end{aligned}
$$

To prove (b), expanding (10) and substituting values from Table 1, we find 


$$
\begin{aligned}
v e-A B C(P N)= & \sqrt{7+8-2 / 7 \times 8}\left(4 m^{2}+2\right)+\sqrt{3+5-2 / 3 \times 5}\left(5 m^{2}-1\right)+\sqrt{5+7-2 / 5 \times 7}\left(18 m^{2}+m+1\right) \\
& +\sqrt{5+5-2 / 5 \times 5}\left(7 m^{2}+1\right) \\
= & \frac{2}{5} \sqrt{2}\left(7 m^{2}+1\right)+\sqrt{2 / 5}\left(5 m^{2}-1\right)+\sqrt{2 / 7}\left(18 m^{2}+m+1\right)+\sqrt{13 / 14}\left(2 m^{2}+1\right) \\
= & \left(\frac{14 \sqrt{2}}{5}+\sqrt{10}+18 \sqrt{2 / 7}+\sqrt{26 / 7}\right) m^{2}+\sqrt{2 / 7} m+\frac{2 \sqrt{2}}{5}-\sqrt{2 / 5}+\sqrt{2 / 7}+\sqrt{13 / 14} \$ .
\end{aligned}
$$

Theorem 4. Let $P N(V, E)$ be the graph of polyphenylene network, then

$$
\begin{aligned}
& \text { (a) } v e-G A(P N)=\left(\frac{16 \sqrt{14}}{15}+\frac{5 \sqrt{15}}{4}+3 \sqrt{35}+7\right) m^{2}+\frac{\sqrt{35} m}{6}+\frac{8 \sqrt{14}}{15}+\frac{\sqrt{35}}{6}-\frac{\sqrt{15}}{4}+1, \\
& \text { (b) } v e-A G(P N)=\left(4 \sqrt{\frac{5}{3}}+\frac{15}{\sqrt{14}}+\frac{108}{\sqrt{35}}+7\right) m^{2}+\frac{6 m}{\sqrt{35}}+\frac{15}{2 \sqrt{14}}-\frac{4}{\sqrt{15}}+\frac{6}{\sqrt{35}}+1 .
\end{aligned}
$$

Proof. To prove (a), expand equation (11) and substitute values from Table 1, which gives

$$
\begin{aligned}
v e-G A(P N) & =\left(4 m^{2}+2\right) \frac{2 \sqrt{7 \times 8}}{7+8}+\left(5 m^{2}-1\right) \frac{2 \sqrt{3 \times 5}}{3+5}+\left(18 m^{2}+m+1\right) \frac{2 \sqrt{5 \times 7}}{5+7}+\left(7 m^{2}+1\right) \frac{2 \sqrt{5 \times 5}}{5+5} \\
& =7 m^{2}+\frac{4}{15} \sqrt{14}\left(4 m^{2}+2\right)+\frac{1}{4} \sqrt{15}\left(5 m^{2}-1\right)+\frac{1}{6} \sqrt{35}\left(18 m^{2}+m+1\right)+1 \\
& =\left(\frac{16 \sqrt{14}}{15}+\frac{5 \sqrt{15}}{4}+3 \sqrt{35}+7\right) m^{2}+\frac{\sqrt{35} m}{6}+\frac{8 \sqrt{14}}{15}+\frac{\sqrt{35}}{6}-\frac{\sqrt{15}}{4}+1 .
\end{aligned}
$$

To prove (b), expand (12) and substitute values from Table 1, which gives

$$
\begin{aligned}
A G^{v e}(P N) & =\left(5 m^{2}-1\right) \frac{3+5}{2 \sqrt{3 \times 5}}+\left(7 m^{2}+1\right) \frac{5+5}{2 \sqrt{5 \times 5}}+\left(18 m^{2}+m+1\right) \frac{7+5}{2 \sqrt{7 \times 5}}+\left(4 m^{2}+2\right) \frac{7+8}{2 \sqrt{7 \times 8}} \\
& =1+7 m^{2}+\frac{15\left(1+2 m^{2}\right)}{2 \sqrt{14}}+\frac{4\left(-1+5 m^{2}\right)}{\sqrt{15}}+\frac{6\left(1+m+18 m^{2}\right)}{\sqrt{35}} \\
& =\left(4 \sqrt{\frac{5}{3}}+\frac{15}{\sqrt{14}}+\frac{108}{\sqrt{35}}+7\right) m^{2}+\frac{6 m}{\sqrt{35}}+\frac{15}{2 \sqrt{14}}-\frac{4}{\sqrt{15}}+\frac{6}{\sqrt{35}}+1 .
\end{aligned}
$$

Theorem 5. Let $P N(V, E)$ be the graph of polyphenylene network; then,

$$
H^{v e}(P N)=\frac{1}{60}\left(371 m^{2}+10 m+23\right)
$$


Proof. To prove, expand equation (13) and substitute values from Table 1, which gives

$$
\begin{aligned}
H^{v e}(P N) & =\frac{2\left(4 m^{2}+2\right)}{7+8}+\frac{2\left(5 m^{2}-1\right)}{3+5}+\frac{2\left(18 m^{2}+m+1\right)}{5+7}+\frac{2\left(7 m^{2}+1\right)}{5+5} \\
& =\frac{2}{15}\left(4 m^{2}+2\right)+\frac{1}{4}\left(5 m^{2}-1\right)+\frac{1}{5}\left(7 m^{2}+1\right)+\frac{1}{6}\left(18 m^{2}+m+1\right) \\
& =\frac{1}{60}\left(371 m^{2}+10 m+23\right) .
\end{aligned}
$$

Theorem 6. Let $P N(V, E)$ be the graph of polyphenylene network; then,

(a) $I D^{e v}(P N)=\frac{1}{15}\left(109 m^{2}+3 m+8\right)$,

(b) $\operatorname{ISI}^{v e}(P N)=\frac{1}{120}\left(1321+350 m+11317 m^{2}\right)$.
Proof. To prove (a), expand equation (14) and substitute values from Table 1, which gives

$$
\begin{aligned}
I D^{e v}(P N) & =\left(5 m^{2}-1\right) \times \frac{1}{4}+\left(7 m^{2}+1\right) \times \frac{1}{4}+\left(18 m^{2}+m+1\right) \times \frac{1}{5}+\left(4 m^{2}+2\right) \times \frac{1}{6} \\
& =\frac{1}{15}\left(109 m^{2}+3 m+8\right) .
\end{aligned}
$$

To prove (b), expand (15) and substitute values from Table 1, which gives

$$
\begin{aligned}
\operatorname{ISI}^{v e}(P N) & =\left(5 m^{2}-1\right) \frac{3 \times 5}{3+5}+\left(7 m^{2}+1\right) \frac{5 \times 5}{5+5}+\left(18 m^{2}+m+1\right) \frac{7 \times 5}{7+5}+\left(4 m^{2}+2\right) \frac{7 \times 8}{7+8} \\
& =\frac{56}{15}\left(2+4 m^{2}\right)+\frac{15}{8}\left(-1+5 m^{2}\right)+\frac{5}{2}\left(1+7 m^{2}\right)+\frac{35}{12}\left(1+m+18 m^{2}\right) \\
& =\frac{1}{120}\left(1321+350 m+11317 m^{2}\right) .
\end{aligned}
$$

Theorem 7. Let $P N(V, E)$ be the graph of polyphenylene network; then,
(a) $F^{e v}(P N)=3882 m^{2}+125 m+557$,
(b) $F^{v e}(P N)=316+74 m+2304 m^{2}$.

Proof. To prove (a), expand equation (16) and substitute values from Table 1, which gives

$$
\begin{aligned}
F^{e v}(P N) & =4^{3}\left(5 m^{2}-1\right)+4^{3}\left(7 m^{2}+1\right)+5^{3}\left(18 m^{2}+m+1\right)+6^{3}\left(4 m^{2}+2\right) \\
& =216\left(4 m^{2}+2\right)+64\left(5 m^{2}-1\right)+64\left(7 m^{2}+1\right)+125\left(18 m^{2}+m+1\right) \\
& =3882 m^{2}+125 m+557 .
\end{aligned}
$$


TABLE 2: The numerical representation of Randic-type indices.

\begin{tabular}{lcccc}
\hline$m$ & $R(\mathrm{PN})$ & $R^{e v}(\mathrm{PN})$ & $R^{v e}(\mathrm{PN})$ & $R R^{e v}(\mathrm{PN})$ \\
\hline 1 & 16.4744 & 17.3938 & 6.8152 & 83.4183 \\
2 & 62.0883 & 64.8895 & 25.7884 & 307.796 \\
3 & 137.839 & 143.751 & 57.2978 & 680.268 \\
4 & 243.727 & 253.978 & 101.343 & 1200.83 \\
5 & 379.753 & 395.571 & 157.925 & 1869.49 \\
6 & 545.915 & 568.529 & 227.043 & 2686.25 \\
7 & 742.214 & 772.853 & 308.697 & 3651.1 \\
8 & 968.651 & 1008.54 & 402.887 & 4764.04 \\
9 & 1225.22 & 1275.6 & 509.613 & 6025.08 \\
10 & 1511.94 & 1574.02 & 628.876 & 7434.21 \\
\hline
\end{tabular}

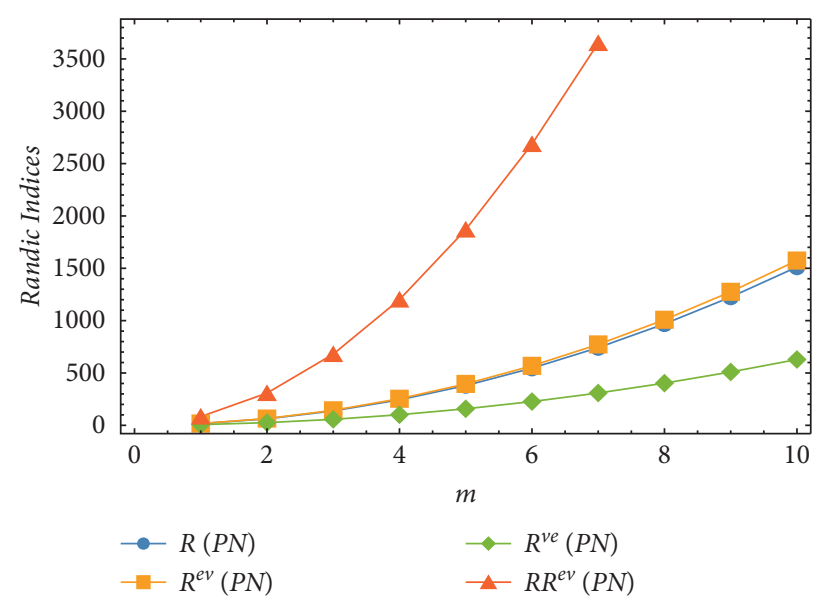

FIgURE 3: The graphical representation of Randic-type indices.

TABLE 3: The numerical representation of Zagreb-type indices.

\begin{tabular}{lcccc}
\hline$m$ & $M_{\mathrm{i}}^{b v e}(\mathrm{PN})$ & $M_{\mathrm{ii}}^{v e}(\mathrm{PN})$ & $R Z G_{\mathrm{iii}}^{v e}(\mathrm{PN})$ & $* M^{v e}(\mathrm{PN})$ \\
\hline 1 & 442 & 1296 & 15920 & 1.71667 \\
2 & 1612 & 4643 & 56150 & 6.5 \\
3 & 3554 & 10198 & 122920 & 14.4456 \\
4 & 6268 & 17961 & 216230 & 39.5533 \\
5 & 9754 & 27932 & 336080 & 57.2556 \\
6 & 14012 & 40111 & 482470 & 77.85 \\
7 & 19042 & 54498 & 655400 & 101.607 \\
8 & 24844 & 71093 & 854870 & 128.526 \\
9 & 31418 & 89896 & 1080880 & 158.607 \\
10 & 38764 & 110907 & 1333430 & \\
\hline
\end{tabular}

To prove (b), expand (17) and substitute values from Table 1, which gives

$$
\begin{aligned}
F^{v e}(P N) & =\left(5 m^{2}-1\right)\left(3^{2}+5^{2}\right)+\left(7 m^{2}+1\right)\left(5^{2}+5^{2}\right)+\left(18 m^{2}+m+1\right)\left(5^{2}+7^{2}\right)+\left(4 m^{2}+2\right)\left(7^{2}+8^{2}\right) \\
& =113\left(2+4 m^{2}\right)+34\left(-1+5 m^{2}\right)+50\left(1+7 m^{2}\right)+74\left(1+m+18 m^{2}\right) \\
& =316+74 m+2304 m^{2} .
\end{aligned}
$$

Theorem 8. Let $P N(V, E)$ be the graph of polyphenylene network; then,
(a) $H M_{1}^{v e}(P N)=6\left(105+24 m+752 m^{2}\right)$,
(b) $\mathrm{HM}_{2}^{v e}(P N)=7897+1225 m+40094 m^{2}$. 


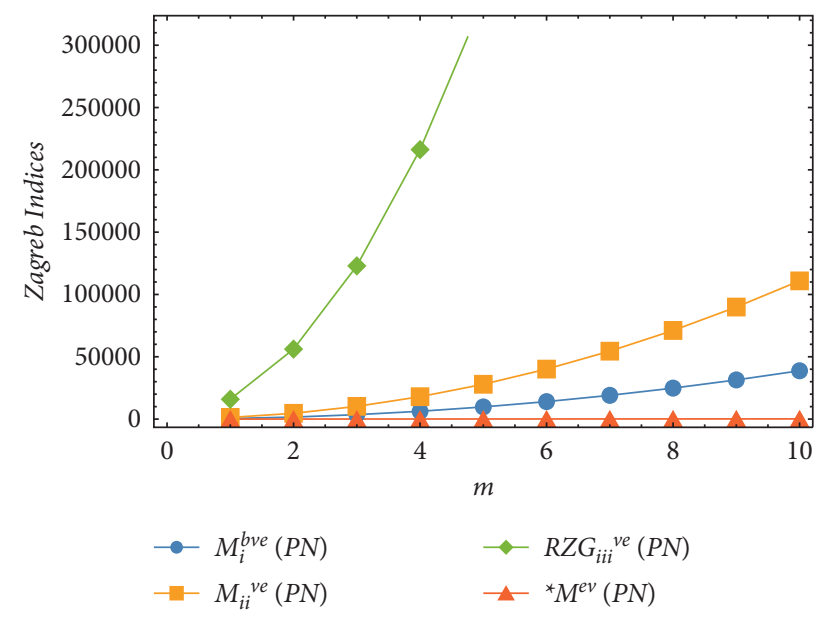

FIgURE 4: The graphical representation of Zagreb-type indices.

TABLE 4: The numerical representation of ve- $\mathrm{ABC}$ and ve-SC indices.

\begin{tabular}{lcc}
\hline$m$ & ve $-\mathrm{ABC}(\mathrm{PN})$ & ve $-\mathrm{SC}(\mathrm{PN})$ \\
\hline 1 & 20.6366 & 11.2667 \\
2 & 77.1833 & 42.1863 \\
3 & 171.072 & 93.5266 \\
4 & 302.301 & 165.287 \\
5 & 470.872 & 257.469 \\
6 & 676.785 & 370.071 \\
7 & 920.039 & 503.094 \\
8 & 1200.63 & 656.537 \\
9 & 1518.57 & 830.401 \\
10 & 1873.85 & 1024.69 \\
\hline
\end{tabular}

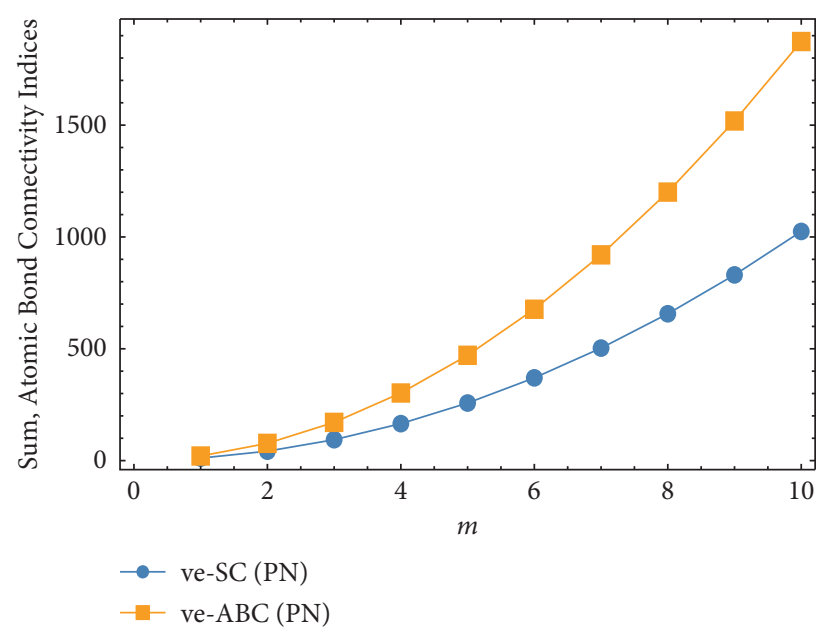

FIgURE 5: The graphical representation of ve-ABC and ve-SC indices.

Proof. To prove (a), expand equation (18) and substitute values from Table 1, which gives

$$
\begin{aligned}
H M_{1}^{v e}(P N) & =\left(5 m^{2}-1\right)(3+5)^{2}+\left(7 m^{2}+1\right)(5+5)^{2}+\left(18 m^{2}+m+1\right)(5+7)^{2}+\left(4 m^{2}+2\right)(7+8)^{2} \\
& =225\left(2+4 m^{2}\right)+64\left(-1+5 m^{2}\right)+100\left(1+7 m^{2}\right)+144\left(1+m+18 m^{2}\right) \\
& =6\left(105+24 m+752 m^{2}\right) .
\end{aligned}
$$


TABLE 5: The numerical representation of the geometric arithmetic index.

\begin{tabular}{lcc}
\hline$m$ & ve - GA(PN) & ve - AG(PN) \\
\hline 1 & 37.5799 & 38.4283 \\
2 & 139.308 & 142.727 \\
3 & 308.196 & 315.882 \\
4 & 544.246 & 557.894 \\
5 & 847.458 & 868.762 \\
6 & 1217.83 & 1248.49 \\
7 & 1655.36 & 1697.07 \\
8 & 2160.06 & 2214.51 \\
9 & 2731.91 & 2800.8 \\
10 & 3370.93 & 3455.95 \\
\hline
\end{tabular}

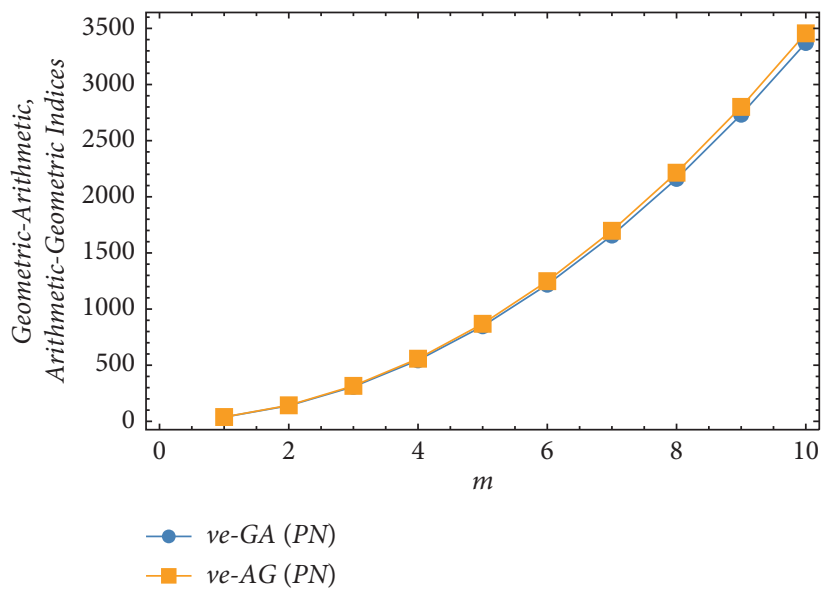

FIgURE 6: The graphical representation of the geometric arithmetic index.

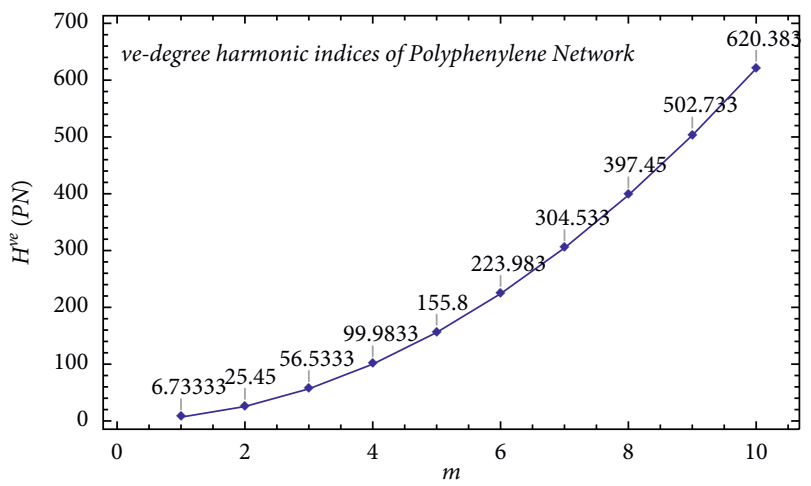

FIGURE 7: The graphical representation of ve-degree harmonic indices.

TABLE 6: The numerical representation of inverse indices.

\begin{tabular}{lcc}
\hline$m$ & $I D^{e v}(\mathrm{PN})$ & $I S I^{v e}(\mathrm{PN})$ \\
\hline 1 & 8 & 108.233 \\
2 & 30 & 394.075 \\
3 & 66.5333 & 868.533 \\
4 & 117.6 & 1531.61 \\
5 & 183.2 & 2383.3 \\
6 & 263.333 & 3423.61 \\
7 & 358 & 4652.53 \\
8 & 467.2 & 6070.07 \\
9 & 590.933 & 7676.23 \\
10 & 729.2 & 9471.01 \\
\hline
\end{tabular}




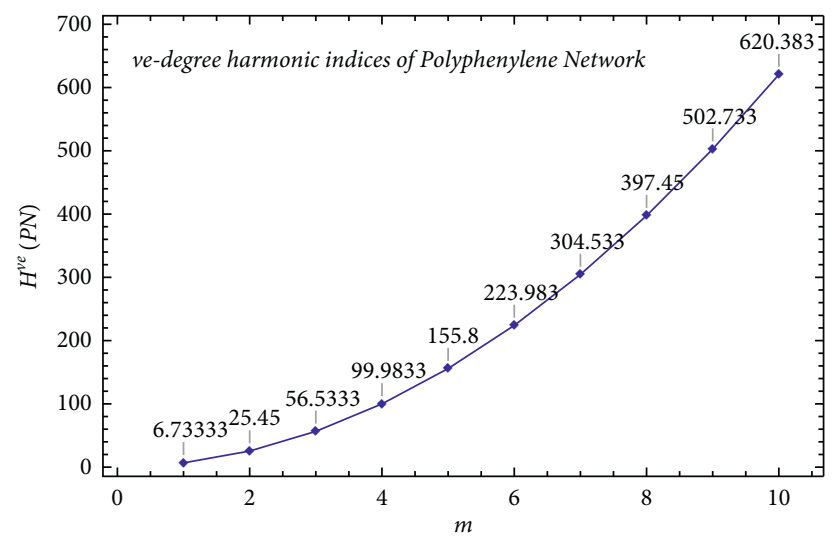

Figure 8: The graphical representation of inverse indices.

TABLE 7: The numerical representation of F-ev-degree index.

\begin{tabular}{lcc}
\hline$m$ & $F^{e v}(\mathrm{PN})$ & $F^{v e}(\mathrm{PN})$ \\
\hline 1 & 4564 & 2694 \\
2 & 16335 & 9680 \\
3 & 35870 & 21274 \\
4 & 63169 & 37476 \\
5 & 98232 & 58286 \\
6 & 141059 & 83704 \\
7 & 191650 & 113730 \\
8 & 250005 & 148364 \\
9 & 316124 & 187606 \\
10 & 390007 & 231456 \\
\hline
\end{tabular}

To prove (b), expand (19) and substitute values from Table 1, which gives

$$
\begin{aligned}
H M_{2}^{v e}(P N) & =\left(5 m^{2}-1\right)(3 \times 5)^{2}+\left(7 m^{2}+1\right)(5 \times 5)^{2}+\left(18 m^{2}+m+1\right)(5 \times 7)^{2}+\left(4 m^{2}+2\right)(7 \times 8)^{2} \\
& =3136\left(2+4 m^{2}\right)+225\left(-1+5 m^{2}\right)+625\left(1+7 m^{2}\right)+1225\left(1+m+18 m^{2}\right) \\
& =7897+1225 m+40094 m^{2}
\end{aligned}
$$

2.2. Numerical and Graphical Discussion. In Theorem 1, we stated and then computed Randić indices of the graph of polyphenylene network (Figure 1) for $m$ frameworks or units. By the resulting equations, we determined exact numerical values of Randić indices, for some $m(=1,2, \ldots, 10)$, and listed in Table 2 and plotted them in Figure 3 by which we can observe that all the resulting equations show parabolic behavior. For continuously increasing values of $m$, all the indices go to infinity. $R R^{e v}(\mathrm{PN})$ increases faster than other indices which are in order $R^{v e}(\mathrm{PN})<R(\mathrm{PN})<R^{e v}(\mathrm{PN})$.

Table 3 and Figure 4 are derived from Theorem 2 which show the numerical values and graphical pattern of the Zagreb indices of polyphenylene network. The curves are parabolic, and the parabola for $R Z G_{\text {iii }}^{v e}(\mathrm{PN})$ has the shortest length of lactusrectum; i.e., it runs much faster than the others.
In Table 4 and Figure 5, we exhibited the numerical measures and graphical style of sum-connectivity and atombond connectivity indices from Theorem 3 . The curves are parabolic, and the separation between them becomes greater and greater for increasing values of $m$.ve - ABC (PN) earns larger numerical value than ve - SC (PN) for all values of $m$.

For $m$ units of polyphenylene, ve $-\mathrm{GA}(\mathrm{PN})$ and ve $\mathrm{AG}(\mathrm{PN})$ were given in Theorem 4. By substituting $m=1, \ldots, 10$, Table 5 is constructed. In Figure 6 , the curves of both the indices are shown from where we can observe that the span between them is growing gradually.

In Figure 7, the outcomes of Theorem 5 are shown. The numerical values are increasing continuously with the increase of frameworks $m$.

Table 6 and Figure 8 are established for Theorem 6 in which we can see that, for all $m, I D^{e v}(\mathrm{PN})<I S I^{v e}(\mathrm{PN})$, and 


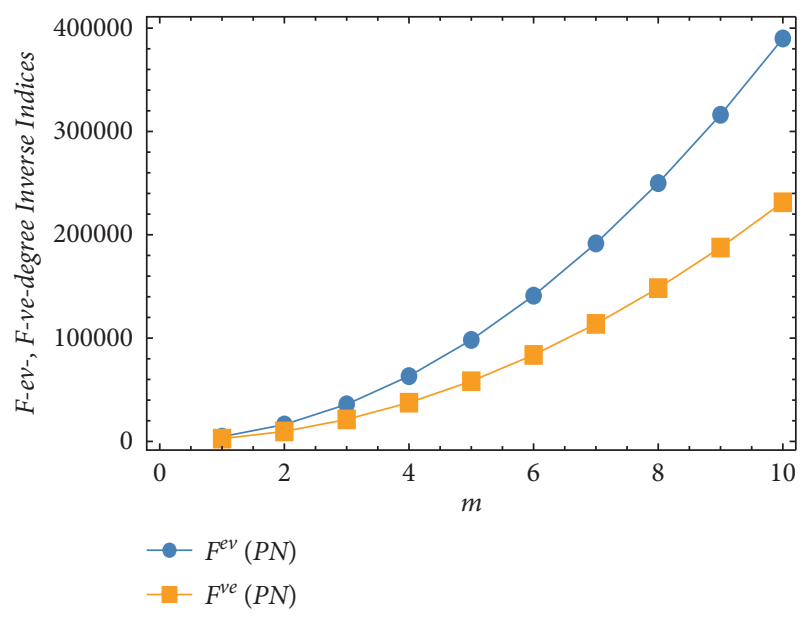

FIgURE 9: The graphical representation of F-ev-degree index.

TABLE 8: The numerical representation of hyper-ve-degree index.

\begin{tabular}{lcc}
\hline$m$ & $H M_{1}^{v e}(P N)$ & $H M_{2}^{v e}(P N)$ \\
\hline 1 & 5286 & 49216 \\
2 & 18966 & 170723 \\
3 & 41670 & 372418 \\
4 & 73398 & 654301 \\
5 & 114150 & $1.01637 \times 10^{6}$ \\
6 & 163926 & $1.45863 \times 10^{6}$ \\
7 & 222726 & $1.98108 \times 10^{6}$ \\
8 & 290550 & $2.58371 \times 10^{6}$ \\
9 & 367398 & $3.26654 \times 10^{6}$ \\
10 & 453270 & $4.02955 \times 10^{6}$ \\
\hline
\end{tabular}

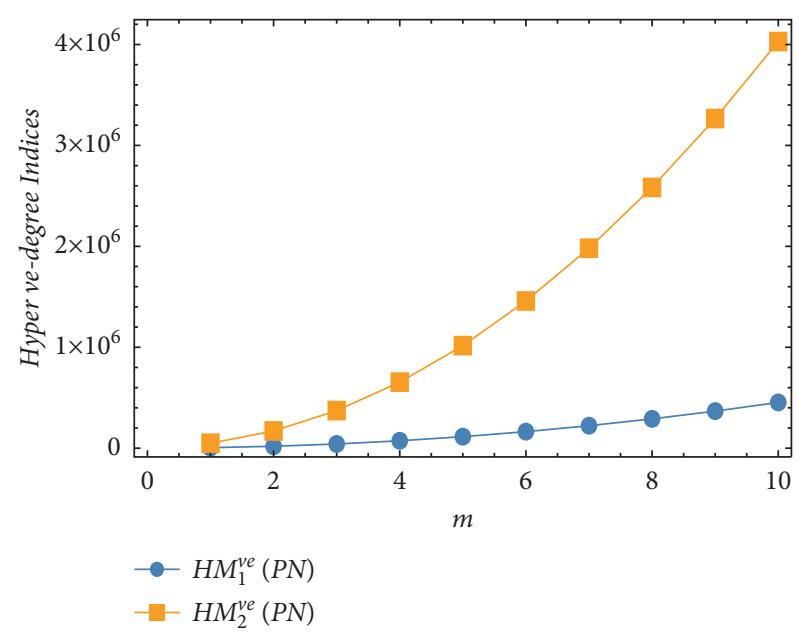

Figure 10: The graphical representation of hyper-ve-degree index.

both the curves are parabolic in manner. The span between the curves is growing very high.

Numerical values of the indices discussed in Theorem 7 are given in Table 7, where the graph of both the indices is shown in Figure 9. Again, both the curve are parabolic. The index $F^{e v}(\mathrm{PN})$ acquires higher values than the index $F^{v e}(\mathrm{PN})$ all the time, for the graph of polyphenylene network.
Following are the conclusions of final Theorem 8. The numerical representation is shown in Table 8 , and graphical representation is shown in Figure 10. Once again both the curves are parabolic. The separation between them is growing continuously. The numerical values of $H M_{1}^{v e}(P N)$ is always less than the numerical values of $H_{M}^{v e}(P N)$.

\section{Conclusion}

In this study, we calculated 19 topological indices discussed in Section 1 for the graph of polyphenylene network. Each index represents a unique chemical or physical property which is also mentioned in section 1 . We also mentioned many qualities of polyphenylene which tell that it is an admirable chemical compound to make new substances and to improve dispositions of the present one. Therefore, the work of this paper will provide assistance in studying the properties of polyphenylene and its derivatives.

\section{Data Availability}

The data used to support the findings of this study are cited at relevant places within the article as references.

\section{Conflicts of Interest}

The authors declare that they have no conflicts of interest.

\section{Authors' Contributions}

All authors equally contributed to this work.

\section{References}

[1] M. Randic, "Nenad Trinajstic pioneer of chemical graph theory," Croatica Chemica Acta, vol. 77, no. 1-2, pp. 1-15, 2004.

[2] I. Gutman, E. Milovanovic, and I. Milovanovic, "Beyond the Zagreb indices," AKCE International Journal of Graphs and Combinatorics, vol. 18, pp. 22-33, 2018.

[3] J. C. Dearden, "The use of topological indices in QSAR and QSPR modeling," in Challenges and Advances in Computational Chemistry and Physics, vol. 14, pp. 57-88, Springer, Cham, 2017.

[4] Y.-M. Chu, M. K. Siddiqui, M. F. Hanif, A. Rauf, M. Ishtiaq, and M. H. Muhammad, "On ve-degree and ev-degree based topological properties of H-naphtalenic nanotube," Polycyclic Aromatic Compounds, vol. 10, pp. 1-13, 2020.

[5] M. Ibrahim, N. Zahra, and M. K. Siddiqui, "On ve degree and ev degree based topological indices for the series of benzenoid graphs," Polycyclic Aromatic Compounds, vol. 8, pp. 1-15, 2021.

[6] M. M. Zobair, M. A. Malik, and H. Shaker, "Eccentricity based topological invariants of tightest nonadjacently configured stable pentagonal structure of carbon nanocones," International Journal of Quantum Chemistry, vol. 121, no. 24, pp. 1-15, 2021.

[7] M. M. Zobair, A. M. Malik, H. Shaker, and N. Rehman, "Eccentricity based topological invariants of triangulane dendrimers," Utilitas Mathematica, vol. 107, pp. 193-206, 2018.

[8] H. Shaker, M. M. Zobair, H. M. A. Mehmood, and M. A. Malik, "Gourava descriptors of multi dimensional flat and stable tri hexagonal boron nanotubes," International Journal of Quantum Chemistry, vol. 122, no. 2, pp. 1-12, 2022. 
[9] M. Chellali, T. W. Haynes, S. T. Hedetniemi, and T. M. Lewis, "On ve-degrees and ev-degrees in graphs," Discrete Mathematics, vol. 340, no. 2, pp. 31-38, 2017.

[10] M. Randic, "Characterization of molecular branching," Journal of the American Chemical Society, vol. 97, no. 23, pp. 6609-6615, 1975.

[11] E. D. . Z. Suleyman, "A new tool for QSPR researches: evdegree randic index," Celal Bayar University Journal of Science, vol. 13, no. 3, pp. 615-618, 2017.

[12] V. R. Kulli, "Computing ev-degree and multiplicative evdegree indices of certain chemical structures," International Journal of Engineering Sciences \& Research Technology, vol. 7, no. 9, pp. 54-65, 2020.

[13] S. Fajtlowicz, "On conjectures of Graffiti-II," Congressus Numerantium, vol. 60, pp. 187-197, 1987.

[14] S. Bajaj, S. S. Sambi, and A. K. Madan, "Prediction of antiinflammatory activity of $\mathrm{N}$-arylanthranilic acids: computational approach using refined Zagreb indices," Croatica Chemica Acta, vol. 78, no. 2, pp. 165-174, 2005.

[15] H. Dureja, S. Gupta, and A. K. Madan, "Topological models for prediction of pharmacokinetic parameters of cephalosporins using random forest, decision tree and moving average analysis," Scientia Pharmaceutica, vol. 76, no. 3, pp. 377-394, 2008.

[16] I. Gutman, J. Tosovic, S. Radenkovic, and S. Markovic, "On atom-bond connectivity index and its chemical applicability," Croatica Chemica Acta, vol. 44, pp. 265-274, 2012.

[17] V. R. Kulli, "On the square ve-degree index and its polynomial of certain oxide networks," Journal of Global Research in Mathematical Archives, vol. 5, no. 10, pp. 1-11, 2018.

[18] D. Vukicevic and B. Furtula, "Topological index based on the ratios of geometrical and arithmetical means of end-vertex degrees of edges," Journal of Mathematical Chemistry, vol. 46, no. 4, pp. 1369-1376, 2009.

[19] B. Zhou and N. Trinajstić, "On general sum-connectivity index," Journal of Mathematical Chemistry, vol. 47, no. 1, pp. 210-218, 2010.

[20] Z. Shao, M. Siddiqui, and M. Muhammad, "Computing zagreb indices and zagreb polynomials for symmetrical nanotubes," Symmetry, vol. 10, no. 7, pp. 244-260, 2018.

[21] V. R. Kulli, "On ve-degree indices and their polynomials of dominating oxide networks," Annals of Pure and Applied Mathematics, vol. 18, no. 1, pp. 1-7, 2018.

[22] Z. Shao, P. Wu, X. Zhang, D. Dimitrov, and J.-B. Liu, "On the maximum $\mathrm{ABC}$ index of graphs with prescribed size and without pendent vertices," IEEE Access, vol. 6, Article ID 27604, 2018.

[23] S. Wang, Z. Shao, J.-B. Liu, and B. Wei, "The bounds of vertex padmakar-ivan index on k-trees," Mathematics, vol. 7, no. 4, pp. 324-334, 2019.

[24] J. B. Liu, S. Wang, C. Wang, and S. Hayat, "Further results on computation of topological indices of certain networks," IET Control Theory \& Applications, vol. 11, no. 13, pp. 2065-2071, 2017.

[25] Y. Li, Z. Zhou, P. Shen, and Z. Chen, "Two-dimensional polyphenylene: experimentally available porous graphene as a hydrogen purification membrane," Chemical Communications, vol. 46, no. 21, pp. 3672-3674, 2010.

[26] W. Gao, M. Siddiqui, M. Naeem, and N. Rehman, "Topological characterization of carbon graphite and crystal cubic carbon structures," Molecules, vol. 22, no. 9, pp. 1496-1515, 2017. 